\title{
Revisiting the analogue of the Jebsen-Birkhoff theorem in Brans-Dicke gravity
}

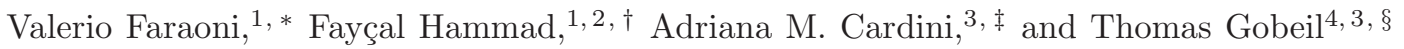 \\ ${ }^{1}$ Department of Physics $\&$ Astronomy and STAR Research Cluster, \\ Bishop's University, 2600 College St., Sherbrooke, Québec, Canada J1M 1Z7 \\ ${ }^{2}$ Physics Department, Champlain College-Lennoxville, \\ 2580 College Street, Sherbrooke, Québec, Canada J1M 0C8 \\ ${ }^{3}$ Department of Physics 83 Astronomy, Bishop's University, \\ 2600 College Street, Sherbrooke, Québec, Canada J1M 1Z7 \\ ${ }^{4}$ Department of Physics, Université de Sherbrooke, \\ 2500 Blvd. de l'Université, Sherbrooke, Québec, Canada J1K 2R1
}

\begin{abstract}
We report the explicit form of the general static, spherically symmetric, and asymptotically flat solution of vacuum Brans-Dicke gravity in the Jordan frame, assuming that the Brans-Dicke scalar field has no singularities or zeros (except possibly for a central singularity). This general solution is conformal to the Fisher-Wyman geometry of Einstein theory and its nature depends on a scalar charge parameter. Apart from the Schwarzschild black hole, only wormhole throats and central naked singularities are possible.
\end{abstract}

\section{INTRODUCTION}

In general relativity (GR), there is a unique spherical and asymptotically flat solution of the vacuum Einstein equations (with zero cosmological constant): the static Schwarzschild geometry. This fact, known as the JebsenBirkhoff theorem [1], generalized in Ref. [2] to higherdimensional GR, is extremely important because it makes GR black holes simple, there is no need to single out the "physical" black hole solutions of GR, and the end point of gravitational collapse is completely determined. The Jebsen-Birkhoff theorem breaks down in theories of gravity alternative to GR, which are motivated by the need to explain the present acceleration of the universe without an ad hoc dark energy, and by unavoidable corrections to GR arising from any attempt to quantize gravity. The prototypical alternative to GR is Brans-Dicke gravity [3], which adds to the metric tensor $g_{a b}$ a scalar degree of freedom $\phi$, approximately corresponding to the inverse of the effective gravitational coupling strength, which becomes dynamical [3]. Brans-Dicke theory was generalized by promoting the constant Brans-Dicke parameter to a function of $\phi$ and/or by including a potential $V(\phi)$ [4]. More modern versions of scalar-tensor gravity include galileons, generalized galileons, and Horndeski theory, which are the subject of intensive research [5]. The vacuum Brans-Dicke action[1]

$$
S_{B D}=\int d^{4} x \frac{\sqrt{-g}}{16 \pi}\left(\phi \mathcal{R}-\frac{\omega}{\phi} \nabla^{c} \phi \nabla_{c} \phi\right)
$$

\footnotetext{
* vfaraoni@ubishops.ca

$\dagger$ fhammad@ubishops.ca

$\ddagger$ a.marina.cardini@gmail.com

$\S$ Thomas.Gobeil@usherbrooke.ca

1 We use units in which the speed of light and Newton's constant are unity, and the notation of Ref. [6].
}

(where $\mathcal{R}$ is the Ricci scalar and $\omega$ is the constant BransDicke coupling) gives rise to the field equations

$$
\begin{aligned}
\mathcal{R}_{a b}-\frac{1}{2} g_{a b} \mathcal{R}= & \frac{\omega}{\phi^{2}}\left(\nabla_{a} \phi \nabla_{b} \phi-\frac{1}{2} g_{a b} \nabla^{c} \phi \nabla_{c} \phi\right) \\
& +\frac{1}{\phi}\left(\nabla_{a} \nabla_{b} \phi-g_{a b} \square \phi\right), \\
\square \phi= & 0 .
\end{aligned}
$$

Black holes in scalar-tensor gravity are not arbitrary: an important no-hair theorem due to Hawking states that all vacuum, stationary, and asymptotically flat black holes of Brans-Dicke gravity must reduce to Kerr black holes 7]. Hawking's no-hair theorem has been generalized to include more general scalar-tensor theories and a potential $V(\phi)$, provided that the latter has a minimum which allows for $\phi$ to sit in a state of equilibrium [8 10]. An essential feature in the proof of no-hair theorems is that $\phi$ becomes constant outside the horizon, reducing the theory to GR and any black hole to Kerr. A few maverick solutions are known which evade the no-hair theorems, but only at the price of physical pathologies such as the divergence of $\phi$ on the horizon (the solution of [11] is one example, but it is linearly unstable [12]). No-hair theorems for Horndeski and galileon theories, and ways to evade them, are the subject of a large literature ([13] and references therein).

In Brans-Dicke theory, things become more tricky. In fact, a theorem due to Agnese and La Camera [14] states that in Brans-Dicke gravity the possible solutions describe only wormholes or naked singularities. Its proof is incorrect, as shown below. In any case, it is clear that this theorem contradicts Hawking's no-hair result and its generalizations [7-10, 15] because it excludes the Schwarzschild black hole which is indeed a solution, as is easy to verify. This Letter aims at presenting a comprehensive report that clarifies the issue by linking it to the most general solution of vacuum Brans-Dicke theory [16], which constitutes an analogue of the Jebsen-Birkhoff the- 
orem of GR in Brans-Dicke theory, reworked here under the following physically reasonable assumptions:

1. the vacuum Brans-Dicke equations in the Jordan frame hold with $\omega \neq-3 / 2$;

2. the spacetime metric is spherically symmetric, static, and asymptotically flat (staticity reflects a state of equilibrium, while asymptotic flatness characterizes isolated objects);

3. the Brans-Dicke scalar $\phi$ depends only on the radial coordinate $r$, it does not have poles or zeros (except possibly for a central singularity), and $\phi(r)$ becomes constant as $r \rightarrow+\infty$.

\section{THE GENERAL JORDAN FRAME SOLUTION}

Let us investigate the general solution under the assumptions above.

\section{A. The Agnese-La Camera theorem}

The Agnese-La Camera theorem [14] states that, under the assumptions 1)-3), the only possible solutions describe wormholes or naked singularities. The proof of this theorem begins by writing the line element and scalar field as

$$
\begin{aligned}
d s_{A L C}^{2}= & -\left(1-\frac{2 \eta}{r}\right)^{A} d t^{2}+\left(1-\frac{2 \eta}{r}\right)^{B} d r^{2} \\
& +\left(1-\frac{2 \eta}{r}\right)^{1+B} r^{2} d \Omega_{(2)}^{2}, \\
\phi_{A L C}(r)= & \phi_{0}\left(1-\frac{2 \eta}{r}\right)^{\frac{-(A+B)}{2}},
\end{aligned}
$$

with $d \Omega_{(2)}^{2}=d \theta^{2}+\sin ^{2} \theta d \varphi^{2}$ and

$$
1-\frac{\omega+1}{\omega+2}=\frac{(A+B)^{2}}{2(1+A B)},
$$

where $A, B$, and $\eta$ are real constants. In [14], this is assumed to be a gauge choice valid for any solution satisfying 1)-3), but at this stage this is instead a choice of a special solution, the Campanelli-Lousto one. The general form of the Campanelli-Lousto solution of Eqs. (2) and (3) is 17

$$
\begin{aligned}
d s_{C L}^{2}= & -\left(1-\frac{2 \eta}{r}\right)^{b_{0}+1} d t^{2}+\left(1-\frac{2 \eta}{r}\right)^{-a_{0}-1} d r^{2} \\
& +\left(1-\frac{2 \eta}{r}\right)^{-a_{0}} r^{2} d \Omega_{(2)}^{2} \\
\phi_{C L}(r)= & \phi_{0}\left(1-\frac{2 \eta}{r}\right)^{\frac{a_{0}-b_{0}}{2}}
\end{aligned}
$$

where $a_{0}$ and $b_{0}$ are two parameters satisfying

$$
\omega=\frac{-2\left(a_{0}^{2}+b_{0}^{2}-a_{0} b_{0}+a_{0}+b_{0}\right)}{\left(a_{0}-b_{0}\right)^{2}} .
$$

It is clear that the Agnese-La Camera choice is reproduced for $a_{0}=-B-1$ and $b_{0}=A-1$. Therefore, the results of [14] are true only for this particular solution (in spite of being advertised as black holes, the CampanelliLousto family contains only wormhole throats and naked singularities [18]). The conflict with the no-hair theorems is then resolved. But what are the solutions satisfying 1)3) which are not Schwarzschild?

\section{B. The general solution}

Let $\left(g_{a b}, \phi\right)$ be a solution under the assumptions 1)-3). By performing the standard conformal transformation to the Einstein frame representation of Brans-Dicke gravity

$$
g_{a b} \rightarrow \tilde{g}_{a b}=\Omega^{2} g_{a b}=\phi g_{a b}
$$

$$
\phi \rightarrow \tilde{\phi}=\sqrt{\frac{|2 \omega+3|}{16 \pi}} \ln \left(\frac{\phi}{\phi_{0}}\right)
$$

(where $\phi_{0}$ is a constant), the Brans-Dicke action (1) is recast in the form

$$
S_{B D}=\int d^{4} x \sqrt{-\tilde{g}}\left(\frac{\tilde{\mathcal{R}}}{16 \pi}-\frac{1}{2} \tilde{g}^{a b} \tilde{\nabla}_{a} \tilde{\phi} \tilde{\nabla}_{b} \tilde{\phi}\right)
$$

Since the conformal factor is $\Omega=\sqrt{\phi(r)}$, the Einstein frame geometry is also spherical, static, and asymptotically flat. Formally, the action (12) describes GR with a free, minimally coupled scalar field and the most general spherical, static, asymptotically flat solution is known to be the Fisher-Janis-Newman-WinicourBuchdahl-Wyman (FJNWBW) solution of GR [19, 20]

$$
\begin{aligned}
d \tilde{s}^{2}= & -\mathrm{e}^{\alpha / r} d t^{2}+\mathrm{e}^{-\alpha / r}\left(\frac{\gamma / r}{\sinh (\gamma / r)}\right)^{4} d r^{2} \\
& +\mathrm{e}^{-\alpha / r}\left(\frac{\gamma / r}{\sinh (\gamma / r)}\right)^{2} r^{2} d \Omega_{(2)}^{2}
\end{aligned}
$$

(where $\alpha$ and $\gamma$ are constants) with scalar field [19, 20]

$$
\tilde{\phi}=\frac{\phi_{*}}{r}, \quad \phi_{*}=\frac{-\sigma}{4 \sqrt{\pi}},
$$

where $\sigma$ is a scalar charge and one can take $\gamma \geq 0$ without loss of generality. These three constants are related by 20]

$$
4 \gamma^{2}=\alpha^{2}+2 \sigma^{2}
$$

If $\sigma=0$, the Einstein frame scalar vanishes, the Jordan frame scalar reduces to a constant, the theory reduces 
to GR, and the solution reduces to Minkowski in both conformal frames, which then coincide. In fact, the constants $\alpha$ and $\gamma$ both vanish whenever $\sigma$ does, thus turning (13) into the Minkowski metric. However, as the notation followed here is that of Ref. 20], the relation (15) between the constants $\alpha, \gamma$ and $\sigma$ does not allow one to see this fact as it only implies $4 \gamma^{2}=\alpha^{2}$ when $\sigma=0$. The vanishing of $\alpha$ and $\gamma$ could be seen only when tracing back the steps that led to expression (13) as presented in Ref. [20], for then one clearly sees that whenever $\sigma$ vanishes, so does the constant $\alpha$, which, in turn, makes $\gamma$ vanish as well.

Consider now the case $\sigma \neq 0$. Mapping the FJNWBW solution back to the Jordan frame, one obtains the most general solution of the Brans-Dicke equations under the assumptions 1)-3) (a remark to this regard was made in passing in [21]). Equation (11) yields the scalar field

$$
\phi(r)=\phi_{0} \mathrm{e}^{-\beta / r}, \quad \beta=\frac{\sigma}{\sqrt{|2 \omega+3|}},
$$

while Eq. (10) gives

$$
\begin{aligned}
d s^{2}= & -\mathrm{e}^{(\alpha+\beta) / r} d t^{2}+\mathrm{e}^{(\beta-\alpha) / r}\left(\frac{\gamma / r}{\sinh (\gamma / r)}\right)^{4} d r^{2} \\
& +\mathrm{e}^{(\beta-\alpha) / r}\left(\frac{\gamma / r}{\sinh (\gamma / r)}\right)^{2} r^{2} d \Omega_{(2)}^{2}
\end{aligned}
$$

This is the most general solution of Brans-Dicke theory under the assumptions 1)-3). It is related to a Campanelli-Lousto solution. The special case $\gamma=0$ will be discussed later.

It should be noted here that in Ref. [16], the most general solution of the generalized Brans-Dicke scalar-tensor theory has also been found by Bronnikov for the case of electrovacuum. For the case of vacuum, explicit forms of the solution corresponding to imaginary $\gamma$, for which the sinh function in the metric (17) is replaced by the sine function, were given there. The latter possibility, corresponding to what has been called in Refs. 22] a "cold black hole", arises for the anomalous case, $2 \omega+3<0$. This case, which we avoided in this paper by taking care of using the absolute value of $2 \omega+3$ in our field redefinition (11), is anomalous for it makes the Einstein frame field $\tilde{\phi}$ imaginary which, in turn, makes the kinetic term in the Einstein frame action (12) acquire the wrong sign. This case gives the ghost counterpart of the solution (13) and (14) due to Bergman and Leipnik [19]. Indeed, when an imaginary field $\tilde{\phi}$ is allowed, the scalar charge $\sigma$ becomes imaginary and the Wyman relation between the various constants becomes [16]

$$
-4 \gamma^{2}=\alpha^{2}-2 \sigma^{2}
$$

The negative signs can be absorbed by letting both $\sigma$ and $\gamma$ be imaginary. This then turns the sinh function into a sine function in (17) 2 In Ref. [23], the special cases $\alpha=$ $\beta, \alpha=(2 \omega+3) \beta$, and $\alpha=-(\omega+1) \beta$ in (17) were found explicitly. Much later, a more exhaustive investigation of the general solutions of the Bergmann-Wagoner class of scalar-tensor theories, in which Brans-Dicke gravity is a special case, was made in Ref. 24]. It was shown there that among these solutions black hole geometries arise for the anomalous versions of these theories. The thermodynamics of such black holes, also dubbed "cold black holes", were investigated.

Let us now come back to our general solution. When $\gamma \neq 0$ in (17), by performing the two consecutive coordinate transformations

$$
\mathrm{e}^{\gamma / r}=\frac{1+B / \rho}{1-B / \rho}, \quad \bar{r}=\rho\left(1+\frac{B}{\rho}\right)^{2},
$$

and setting $\eta=2 B=\sqrt{m^{2}+\sigma^{2}}, m / \eta=-\alpha /(2 \gamma)$, $\sigma / \eta=\beta \sqrt{|2 \omega+3|} /(2 \gamma)$, and rescaling the time coordinate by a factor $|\gamma /(2 B)|$, the solution (17), (16) becomes

$$
\begin{aligned}
d s^{2}= & -\left(1-\frac{2 \eta}{\bar{r}}\right)^{\frac{1}{\eta}\left(m-\frac{\sigma}{\sqrt{|2 \omega+3|}}\right)} d t^{2} \\
& +\left(1-\frac{2 \eta}{\bar{r}}\right)^{\frac{-1}{\eta}\left(m+\frac{\sigma}{\sqrt{|2 \omega+3|}}\right)} d \bar{r}^{2} \\
& +\left(1-\frac{2 \eta}{\bar{r}}\right)^{1-\frac{1}{\eta}\left(m+\frac{\sigma}{\sqrt{|2 \omega+3|}}\right)} \bar{r}^{2} d \Omega_{(2)}^{2}, \\
\phi= & \phi_{0}\left(1-\frac{2 \eta}{\bar{r}}\right)^{\frac{\sigma}{\eta \sqrt{|2 \omega+3|}}},
\end{aligned}
$$

which is a Campanelli-Lousto solution with

$$
\begin{aligned}
& a_{0}=-1+\frac{1}{\eta}\left(m+\frac{\sigma}{\sqrt{|2 \omega+3|}}\right), \\
& b_{0}=-1+\frac{1}{\eta}\left(m-\frac{\sigma}{\sqrt{|2 \omega+3|}}\right) .
\end{aligned}
$$

It must be noted here that although this form contains only the absolute value of the term $2 \omega+3$, the anomalous case $2 \omega+3<0$ discussed above does not apply here as the coordinate redefinitions (19) would not be real-valued anymore since $\gamma$ is imaginary in this case. Therefore, the Campanelli-Lousto metric (7), as well as its other version (44) used in Ref. [14], are only valid for the normal case $2 \omega+3>0$.

\footnotetext{
${ }^{2}$ For completeness, we give here the general solution of BransDicke theory in the anomalous case. It reads,

$d s^{2}=-\mathrm{e}^{\frac{\alpha+\beta}{r}} d t^{2}+\mathrm{e}^{\frac{\beta-\alpha}{r}}\left(\frac{\gamma / r}{\sin (\gamma / r)}\right)^{2}\left[\left(\frac{\gamma / r}{\sin (\gamma / r)}\right)^{2} d r^{2}+r^{2} d \Omega_{(2)}^{2}\right]$.
} 


\section{Generality of the solution}

It seems that, given a solution $\left(g_{a b}, \phi\right)$ of the form (20), (21), one could still change the scalar field according to $\phi \rightarrow \Phi=\phi \mathrm{e}^{\psi(r)}$ in such a way that $\left(g_{a b}, \Phi\right)$ is still a solution, which would mean that (17), (16) do not give all the possible solutions, and hence do not constitute the most general solution. We show here that this is not the case. In fact, the Ricci tensor component

$$
R_{r r}=\omega\left(\frac{\nabla_{r} \phi}{\phi}\right)^{2}+\frac{\nabla_{r} \nabla_{r} \phi}{\phi}
$$

does not change when $\left(g_{a b}, \phi\right)$ is changed into $\left(g_{a b}, \Phi\right)$ provided that

$$
(\omega+1)\left(\nabla_{r} \psi\right)^{2}+2(\omega+1) \frac{\nabla_{r} \phi}{\phi} \nabla_{r} \psi+\nabla_{r} \nabla_{r} \psi=0,
$$

while it must be $\square\left(\phi \mathrm{e}^{\psi}\right)=0$ in order for $\Phi$ to still be a solution. Using this equation to eliminate $\nabla_{r} \nabla_{r} \psi$ in Eq. (25), one finds

$$
\left(\nabla_{r} \psi+\frac{2 \nabla_{r} \phi}{\phi}\right) \nabla_{r} \psi=0
$$

which (apart from the irrelevant possibility $\psi=$ const.) integrates to $\psi(r)=-2 \ln \phi+$ const. and $\Phi=C_{0} / \phi$. However, replacing $\left(g_{a b}, \phi\right)$ with $\left(g_{a b}, C_{0} / \phi\right)$ amounts to changing the exponent $\beta$ in Eq. (16) into $-\beta$, or to changing the sign of the scalar charge $\sigma$, a possibility already included in the form of the general solution (17), (16).

It must be noted, however, that what makes the conformal transformation one to one and protects (17) and (16) against such redefinitions as $\phi \longrightarrow \phi e^{\psi(r)}$, that could have prevented them from being the most general solution, is the homogeneous wave equation $\square \phi=0$. The latter, in turn, is always guaranteed to hold in vacuo and electrovacuo for which the matter energy-momentum tensor is traceless. Therefore, we conclude that (17) and (16) constitute indeed the most general solution with the assumptions 1)-3) above. Moreover, this analysis also applies, and therefore reinforces, Bronnikov's general solutions for vacuum and electrovacuum scalar-tensor theories found in Ref. [16].

\section{Nature of the solution}

In this subsection we investigate the nature of the general solution (17) and (16). To assess whether the general geometry (17) describes black holes, wormholes, or naked singularities, one examines the horizons (if they exist) and their nature. The equation we are going to use for locating the apparent horizons is [25, 26] $\nabla^{c} R \nabla_{c} R=0$, where

$$
R(r)=\gamma \frac{\mathrm{e}^{\frac{\beta-\alpha}{2 r}}}{\sinh (\gamma / r)}
$$

is the areal radius. Horizons correspond to the roots of that equation; a single root describes a black hole horizon while a double root describes a wormhole throat. With (27), the equation becomes

$$
g^{r r}\left(\frac{d R}{d r}\right)^{2}=\sinh ^{2}(\gamma / r)\left[\frac{\alpha-\beta}{2 \gamma}+\operatorname{coth}(\gamma / r)\right]^{2}=0 .
$$

It is clear that, if roots exist, they are always double roots corresponding to wormhole throats. They exist if $(\beta-\alpha) / \gamma>0$ and, in this case, they are given by

$$
r_{H}=\frac{2 \gamma}{\ln \left(\frac{\beta-\alpha+2 \gamma}{\beta-\alpha-2 \gamma}\right)}=\frac{\gamma}{\tanh ^{-1}\left(\frac{2 \gamma}{\beta-\alpha}\right)},
$$

If $(\beta-\alpha) / \gamma<0$, instead, there is a naked singularity. In fact, the general solution (17) has a spacetime singularity at $R=0$, as is deduced from the Ricci scalar

$$
\mathcal{R}=\frac{\omega}{\phi^{2}} \nabla^{c} \phi \nabla_{c} \phi=\left\{\begin{array}{cl}
\frac{\omega \beta^{2}}{\gamma^{4}} \mathrm{e}^{(\alpha-\beta) / r} \sinh ^{4}(\gamma / r) & \text { if } \gamma \neq 0, \\
\frac{\omega \beta^{2}}{r^{4}} \mathrm{e}^{(\alpha-\beta) / r} & \text { if } \gamma=0,
\end{array}\right.
$$

If $\gamma \neq 0$ then when $r \rightarrow 0$ we have, depending on whether $\gamma$ is positive or negative,

$$
\mathcal{R}=\frac{\omega \beta^{2}}{16 \gamma^{4}} \mathrm{e}^{(\alpha-\beta \pm 4 \gamma) / r},
$$

respectively. Therefore, the Ricci scalar diverges as $r \rightarrow 0$ only for $\beta-\alpha<4 \gamma$ or for $\alpha-\beta>4 \gamma$, respectively.

In the special case $\gamma=0$, the FJNWBW metric reduces to the Yilmaz geometry 27] and its Jordan frame cousin (17), (16) is the Brans Class IV solution 28]

$$
\begin{aligned}
d s^{2} & =-\mathrm{e}^{-2 B / r} d t^{2}+\mathrm{e}^{2 B(C+1) / r}\left(d r^{2}+r^{2} d \Omega_{(2)}^{2}\right), \\
\phi & =\phi_{0} \mathrm{e}^{-B C / r},
\end{aligned}
$$

where $B=-(\alpha+\beta) / 2, C=-2 \beta /(\alpha+\beta)$. The equation locating the apparent horizons reduces to $\left(1-\frac{\beta-\alpha}{2 r}\right)^{2}=$ 0 , which has a double root $r_{H}=(\beta-\alpha) / 2$ corresponding to a wormhole throat if $\beta>\alpha$ and to a central naked singularity otherwise.

These results about the nature of the solutions of Brans-Dicke theory have already been worked out in detail in [29]. Therefore, this analysis satisfactorily shows that it is possible to use the most general solution (17) of Brans-Dicke theory to recover in a compact way the results already found in Ref. [29] by going through each of the Brans classes of solutions individually. The general solution (17) thus allows for a unified investigation of the physics behind the four Brans classes of solutions.

It must be noted here that, just as it was done in Ref. [29], the investigation of the nature of the solution conducted here is based on the simple detection of possible black hole horizons or wormhole throats. In fact, 
in contrast to the analysis made in Ref. [30], no additional requirements, such as asymptotic flatness or regularity of the spacetime away from the throat when the latter exists, are imposed before calling such a solution a wormhole. The wormhole definition that is implicitly adopted here, and which was also adopted in Ref. 29], is that of Ref. [31] which consists of a quasi-local definition involving only the properties of the local geometry of spacetime. Of course, "extended" wormholes can also be studied, and they are related to the "cold black holes" of 22]. We refer the reader to 22] for these situations.

\section{CONCLUSIONS}

The key to solving the Brans-Dicke equations under the assumptions 1)-3) is to map the problem into the Einstein frame and using a known result of Einsteinmassless Klein-Gordon theory [19]. By contrast, little progress is made when analyzing directly the Jordan frame field equations. The previous section shows that the Schwarzschild black hole is obtained when the scalar charge $\sigma$ vanishes and that there is no other black hole solution under the assumptions made. This result matches the no-hair theorems of [7-10, 15] (which are, however, more general). The remaining solutions, corresponding to $\sigma \neq 0$, are necessarily of the CampanelliLousto form (7), (8) or conformal to it. They can only describe wormhole throats or naked singularities, according to the values of the parameters $\sigma / m$ and $\omega$ (or of $\alpha$ and $\beta$ ).

The most general solution of Jordan frame Brans-Dicke theory under the assumptions 1)-3) is given by Eqs. (17) and (16) and is conformal to the FJNWBW solution of GR. Our analysis in Sec. IIC established the general character of this solution and pointed to the homogeneous wave equation that the scalar field obeys as being the ingredient that renders the solution (17) really general. We also pointed out that it is only thanks to this constraint that the conformal transformation trick remains a one-to-one mapping and allows one to extract the general Jordan frame solution from the most general Einstein frame one. The homogeneity of the wave equation, being guaranteed by the tracelessness of the matter energy-momentum tensor, makes the conformal trick work both in vacuum and electrovacuum. The conformal transformation trick is indeed what has allowed Bronnikov in Ref. [16] to extract the general solution for the electrovacuum case as well.

We have investigated the general solution of vacuum Brans-Dicke gravity, a result that constitutes an analogue of the Jebsen-Birkhoff theorem of GR in BransDicke theory, which is a rarity in alternative gravities. In principle, this result can be circumvented in the same ways already conceived to evade the no-hair theorems for scalar-tensor black holes: by including matter, by allowing the scalar field to depend on time while keeping the geometry static, or by letting the scalar field diverge or vanish on the horizons 13 .

As already noted above, generalizations of the results presented here have already been obtained. The generalization of the Jebsen-Birkhoff theorem to multidimensional GR was given in Ref. [2], and the general spherically symmetric solution of the Bergmann-Wagoner class of scalar-tensor theories, of which Brans-Dicke gravity is a special case, has been found in Ref. [24]. Generalizations to situations in which a cosmological constant or a non-gravitational scalar field are present and, more important, to axial symmetry, may be possible and will be explored elsewhere.

Acknowledgments: The authors are grateful to K.A. Bronnikov for bringing to their attention Refs. [2, 16, 24], to N. Van Den Bergh for communicating Ref. [23], and to an anonymous referee for useful comments and references. This work is supported by the Natural Sciences and Engineering Research Council of Canada (Grants No. 2016-03803 and No. 2017-05388).
[1] J.T. Jebsen, Ark. Mat. Ast. Fys. (Stockholm) 15, nr. 18 (1921), reprinted in Gen. Relat. Gravit. 37, 2253 (2005); G.D. Birkhoff, Relativity and Modern Physics (Harvard University Press, Cambridge, 1923), p. 253.

[2] K. A. Bronnikov and V. N. Melnikov, Gen. Rel. Gravit. 27, 465 (1995).

[3] C.H. Brans and R.H. Dicke, Phys. Rev. 124, 925 (1961).

[4] P.G. Bergmann, Int. J. Theor. Phys. 1, 25 (1968); R.V. Wagoner, Phys. Rev. D 1, 3209 (1970); K. Nordvedt, Astrophys. J. 161, 1059 (1970); K. Nordtvedt, Phys. Rev. 169, 1017 (1968).

[5] T. Clifton, P.G. Ferreira, A. Padilla, and C. Skordis, Phys. Reps. 513, 1 (2012).

[6] R.M. Wald, General Relativity (Chicago University Press, Chicago, 1984).

[7] S.W. Hawking, Commun. Math. Phys. 25, 167 (1972).

[8] J. D. Bekenstein, arXiv:gr-qc/9605059.
[9] T.P. Sotiriou and V. Faraoni, Phys. Rev. Lett. 108, 081103 (2012).

[10] S. Bhattacharya, K.F. Dialektopoulos, A.E. Romano, and T.N. Tomaras, Phys. Rev. Lett. 115, 181104 (2015).

[11] N. Bocharova, K. Bronnikov, and V. Melnikov, Vestn. Mosk. Univ. Fiz. Astron. 6, 706 (1970).

[12] K.A. Bronnikov and Yu.N. Kireyev, Phys. Lett. A 67, 95 (1978).

[13] J.D. Bekenstein, in Second International A.D. Sahkarov Conference on Physics, Moscow, 20-24 May 1996, edited by I.M. Dremin and A.M. Semikhatov (World Scientific, Singapore, 1997) [arXiv:9605059]; T.P. Sotiriou and S.-Y. Zhou, Phys. Rev. Lett. 112, 251102 (2014); T.P. Sotiriou, Class. Quantum Grav. 32, 214002 (2015); C.A.R. Herdeiro and E. Radu, Int. J. Mod. Phys. D 24, 1542014 (2015); E. Babichev and C. Charmousis, J. High Energy Phys. 08, 106 (2014). 
[14] A.G. Agnese and M. La Camera, Phys. Rev. D 51, 2011 (1995).

[15] V. Faraoni, Phys. Rev. D 95, 124013 (2017).

[16] K.A. Bronnikov, Acta Phys. Polon. B 4, 251 (1973).

[17] M. Campanelli and C. Lousto, Int. J. Mod. Phys. D 02, 451 (1993).

[18] L. Vanzo, S. Zerbini, and V. Faraoni, Phys. Rev. D 86, 084031 (2012).

[19] I.Z. Fisher, Zh. Eksp. Teor. Fiz. 18, 636 (1948) arXiv:gr-qc/9911008; O. Bergman and R. Leipnik, Phys. Rev. 107, 1157 (1957); A.I. Janis, E.T. Newman, and J. Winicour, Phys. Rev. Lett. 20, 878 (1968); H.A. Buchdahl, Int. J. Theor. Phys. 6, 407 (1972);

[20] M. Wyman, Phys. Rev. D 24, 839 (1981).

[21] A. Bhadra and K. Sarkar, Gen. Relat. Gravit. 37, 2189 (2005).

[22] K.A. Bronnikov, C.P. Constantinidis, R.L. Evangelista, and J.C. Fabris, UFES-DF-001/97, arXiv:gr-qc/9710092; K.A. Bronnikov, G. Clément,
C.P. Constantinidis, and J.C. Fabris, Phys. Lett. A 243, 121 (1998).

[23] N. Van Den Bergh, Gen. Rel. Gravit. 12, 863 (1980).

[24] K.A. Bronnikov, C.P. Constantinidis, R.L. Evangelista, and J.C. Fabris, Int. J. Mod. Phys. D 8, 481 (1999).

[25] C.W. Misner and D.H. Sharp, Phys. Rev. 136, B571 (1964).

[26] A.B. Nielsen and M. Visser, Class. Quantum Grav. 23, 4637 (2006).

[27] H. Yilmaz, Phys. Rev. 111, 1417 (1958).

[28] C.H. Brans, Phys. Rev. 125, 2194 (1962).

[29] V. Faraoni, F. Hammad, and S.D. Belknap-Keet, Phys. Rev. D 94, 104019 (2016).

[30] K.A. Bronnikov, M.V. Skvortsova, and A.A. Starobinsky, Gravit. Cosmol. 16, 216 (2010).

[31] D. Hochberg and M. Visser, Phys. Rev. D 58, 044021 (1998). 